\title{
A Comt1 Loss of Function Mutation Is Insufficient for Loss of Pungency in Capsicum
}

\author{
Sota Koeda1,2, Kosuke Sato ${ }^{2}$, Yuri Tanaka², Rihito Takisawa², Akira Kitajima² \\ ${ }^{1}$ Faculty of Agriculture, Kinki University, Nara, Japan \\ ${ }^{2}$ Experimental Farm, Graduate School of Agriculture, Kyoto University, Osaka, Japan \\ Email: 818sota@nara.kindai.ac.jp
}

Received 16 April 2015; accepted 23 May 2015; published 26 May 2015

Copyright @ 2015 by authors and Scientific Research Publishing Inc.

This work is licensed under the Creative Commons Attribution International License (CC BY). http://creativecommons.org/licenses/by/4.0/

(c) (i) Open Access

\section{Abstract}

The participation of $O$-methyltransferase (COMT) in phenylpropanoid-mediated capsaicinoid biosynthesis has long been proposed. Ferulic acid, a phenylpropanoid intermediate, is a precursor of capsaicinoid biosynthesis and is produced from caffeic acid by the action of COMT. As previously reported that silencing Comt expression caused a drastic decrease in capsaicinoid accumulation, it was presumed that a Comt loss-of-function mutation would cause loss of pungency in Capsicum. This hypothesis was tested by cloning Comt1 and Comt 2 from the placenta tissue of the pungent cultivar Habanero. The phylogenetic analysis and comparison of critical amino-acid residues for enzyme function showed that the two COMTs had high similarity with the COMTs of other plant species. Moreover, as the two Comts were both expressed in placenta tissue and expressed prior to the accumulation of capsaicinoids, the two genes could be candidates for capsaicinoid biosynthesis. Second, Comt1 loss-of-function mutants were screened from the germplasm. A truncated Comt1 transcript was expressed in non-pungent pepper No.3341 caused by deletion of the genomic region. The predicted No.3341 COMT1 lacked His-265, which was absolutely necessary for enzymatic activity. Contrary to our expectations, the Comt1 mutation was not related to non-pungency of No.3341, as the deletion of Comt 1 did not co-segregate with non-pungency in the $F_{2}$ population obtained from crossing No.3341 with Habanero. This result was confirmed by screening several pungent accessions harboring the same Comt1 deletion mutation. Although the participation of COMT in phenylpropanoid-mediated capsaicinoid biosynthesis has long been proposed, our present study shows that Comt1 can not be a target for controlling fruit pungency.

\section{Keywords}

Capsaicinoids, Capsicum, Comt, Pepper, Phenylpropanoid Pathway, Pungency 


\section{Introduction}

Capsicum, a member of the family Solanaceae, originated from and was first domesticated in South and Central Americas [1]. Based on archeological evidence, Capsicum was already domesticated in 6000 B.P., making it one of the earliest domesticated plant genera [2]. Capsicum was introduced to Europe at the end of the 15th century, after the first voyage of Christopher Columbus, and its use spreads rapidly over the Old World continents. Because Capsicum accumulates pungent capsaicinoid analogs in its fruit, it is one of the most important spices used worldwide. Moreover, as non-pungent cultivars, such as bell pepper or paprika, contain a high amount of vitamins, Capsicum is also an important vegetable. Thus, to understand how capsaicinoids are biosynthesized is important, as both pungent and non-pungent peppers are valuable targets for spice and vegetable breeding.

Capsaicinoids are synthesized in placenta tissue from L-phenylalanine through the phenylpropanoid pathway to extract cinnamic, $p$-coumaric, caffeic and ferulic acid followed by vanillin and vanillylamine, which is ultimately linked to the branched fatty acid residues (8-methyl-6-nonenoic, 8-methylnonanoic, 7-methyloctanoic, 9methyl-6-decenoic, or 9-methyldecanoic acid) that are synthesized from L-valine or L-leucine to give five capsaicinoid analogs: capsaicin, dihydrocapsaicin, nordihydrocapsaicin, homocapsaicin, and homodihydrocapsaicin [3]-[6] (Figure 1). The general capsaicinoid biosynthetic pathway has been studied for almost half a century, and transcripts expressed in placenta tissue of pungent cultivars have been isolated [7]-[9].

A single genetic source for non-pungency has been suggested by the early identification in the 1500s of a widely distributed non-pungent C. annuum pepper [10], now known to carry a recessive acyltransferase (Pun1) allele [11]. Most of the non-pungent cultivars within C. annuum carry the same single Pun1 recessive allele [11]. Two recessive putative aminotransferase ( $p$-AMT) alleles have been reported in the $\mathrm{CH}-19$ Sweet and Himo nonpungent cultivars [12] [13]. In addition, a single recessive Pun1 allele has been reported for $C$. chinense, $C$. frutescens, and C. chacoense [14], and four recessive $p$-AMT alleles have been reported for C. chinense [15] [16]. Although the capsaicinoid biosynthetic pathway involving specific enzymes and genes has been proposed,

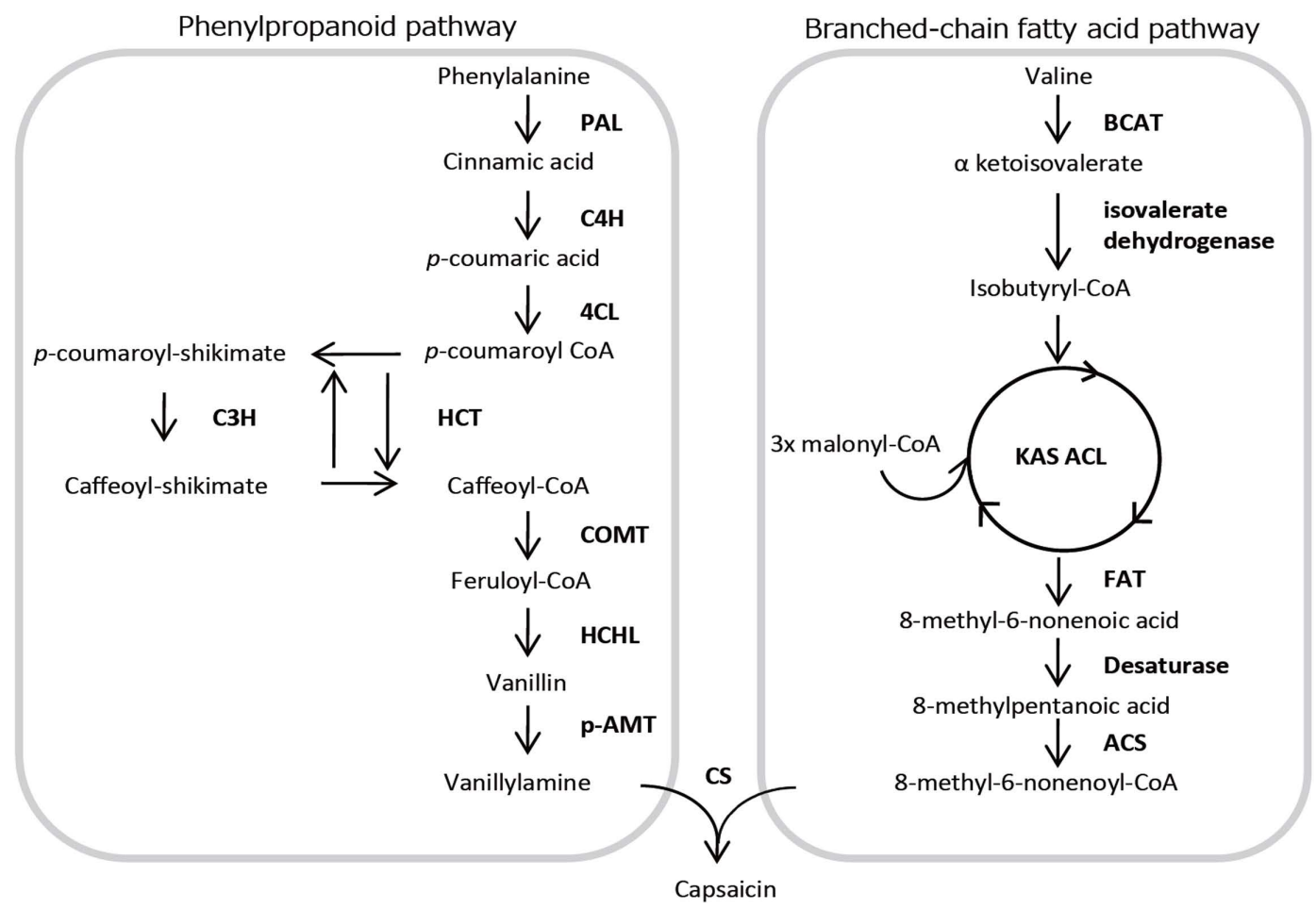

Figure 1. Capsaicinoid biosynthetic pathway (modified from Stewart et al. [11]). PAL, phenylalanine ammonia-lyase; C4H, cinnamic acid 4-hydroxylase; 4CL, 4-coumarate-CoA ligase; HCT, hydroxycinnamoyl transferase; $\mathrm{C} 3 \mathrm{H}$, coumaroyl shikimate/quinate 3-hydroxylase; COMT, caffeic acid O-methyltransferase; HCHL, hydroxycinnamoyl-CoA hydratase lyase; p-AMT, putative aminotransferase; BCAT, branched-chain amino acid transferase; KAS, $\beta$-ketoacyl-[acyl-carrier-protein] (ACP) synthase; ACL, acyl carrier protein; FAT, acyl-ACP thioesterase; ACS, acyl-CoA synthase; CS, capsaicin synthase. 
and some transcripts for those genes specifically accumulate in placenta tissue, except for Pun1 and $p$-AMT, no direct evidence of their participation in capsaicinoid production has been reported.

Abraham-Juarez et al. [6] silenced Comt, 3-keto-acyl-ACP synthase (Kas), and p-AMT by virus induced gene silencing (VIGS) and showed that the accumulation of capsaicinoids decreased drastically. This result indicates that a loss-of-function mutation in Comt or Kas may induce loss of pungency in Capsicum. Ferulic acid is a precursor of capsaicinoid biosynthesis and is produced from caffeic acid by the action of COMT [5] [7] [17] [18]. COMT is one of the S-adenosyl-L-methionine (SAM)-dependent $O$-methyltransferases (OMTs) that methylate small molecules involved in the biosynthesis of lignin, flavonoids, alkaloids, and many other plant secondary products [19]. The $O$-methylation patterns of polyhydroxylated small molecules are crucial in plants to determine final product distribution via multiple branched biosynthetic pathways using the same or similar intermediates and substrates [20]. Two OMTs expressed in Capsicum placenta tissue, CaOMT1 (U83789.1) from C. annuum [21] and Comt (AF081214.1) from C. chinense [7] have been cloned. Although the participation of Comt in phenylpropanoid-mediated capsaicinoid biosynthesis has long been proposed, it is still unclear whether Comt could be a target for controlling fruit pungency.

The objectives of the present study were 1) to clarify whether the two previously cloned OMTs were allelic or different genes coded at different loci, 2) to investigate whether the Comt loss-of-function mutation caused loss of pungency in Capsicum. To this end, two OMTs were cloned from placenta tissue of Habanero (C. chinense). In addition, phylogenetic and expression analyses of the two OMTs were conducted. Moreover, natural deletion mutants of one of the OMTs that had high similarity to Comt (AF081214.1) were screened from the germplasm and the phenotype were analyzed for pungency.

\section{Materials and Methods}

\subsection{Plant Materials}

C. chinense cultivars Habanero, No.3341, No.3582, No.3614, No.3616, No.3628, No.3639, No.3641, No.3643, No.3645, and No.3646 were used. An inbred line of Habanero was prepared by self-fertilizing six times. $F_{1}$ and $\mathrm{F}_{2}$ progeny were obtained by crossing Habanero with No.3341. All plants were grown on the Kyoto University Experimental Farm, from March to October 2012 and 2013.

\subsection{Phenotyping Fruit Pungency}

After the fruits were freeze-dried, the capsaicinoids were extracted and quantified according to the method described by Koeda et al. [16]. Capsaicinoid content was calculated as the sum of capsaicin and dihydrocapsaicin.

\subsection{Comt cDNA Sequence Analysis}

Pepper fruits were harvested at three stages, and the placenta was separated for RNA extraction. Total RNA was extracted and reverse transcribed according to the method described by Koeda et al. [22]. CaActin (AY572427) was used as a positive internal control for reverse transcription-polymerase chain reaction (RT-PCR). The partial Comt 1 cDNA sequence was amplified using Comt1-F and Comt-R1 primer sets, and Comt2 was amplified using Comt2-F and Comt-R1 primer sets (Table 1). The full-length Comt1 cDNA sequence was amplified using Comt1-F and Comt1-R primer sets, and Comt2 was amplified using Comt2-F and Comt2-R primer sets (Table 1). A Gene Racer kit (Invitrogen, Carlsbad, CA, USA) was used to clone truncated Comt1 of No.3341. PCR was performed using KOD Plus Neo (Toyobo, Osaka, Japan). The reaction mixtures for all PCR reactions were initially denatured at $94^{\circ} \mathrm{C}$ for $2 \mathrm{~min}$, followed by 35 cycles at $94^{\circ} \mathrm{C}$ for $30 \mathrm{~s}, 60^{\circ} \mathrm{C}$ for $30 \mathrm{~s}$, and $72^{\circ} \mathrm{C}$ for 2 min, terminating with a $3 \mathrm{~min}$ extension at $72^{\circ} \mathrm{C}$. Electrophoresis using $1.0 \%(\mathrm{w} / \mathrm{v})$ agarose gels was performed on the amplified PCR products. Three biological replicates of RT-PCR were performed for all treatments using independently prepared total RNA, and similar results were obtained. The full-length Comt 1 and Comt2 sequences amplified by RT-PCR were cloned into the pTaq1 cloning vector (BioDynamics Laboratory, Tokyo, Japan). Nucleotide sequencing was performed in an ABI PRISM 3100 genetic analyzer with an ABI PRISM BigDye Terminator v3.1 Cycle Sequencing Kit (Applied Biosystems, Foster City, CA, USA).

\subsection{Comt Genomic Sequence Analysis}

Genomic DNA was extracted from young leaves of pepper plants using Nucleon PhytoPure (GE Healthcare, 
Table 1. Primers used in this study.

\begin{tabular}{ccc}
\hline Primer name & Sequence (5'-3') & Tm $\left({ }^{\circ} \mathrm{C}\right)$ \\
\hline Comt1-F & TTTCCGAATTCAGTTATTCAAACA & 60 \\
Comt1-R & TCAATAGAGATTCTGGTTTTCTCA & 60 \\
Comt2-F & TTTCTTCCCTTAGCTTGCCTAAT & 60 \\
Comt2-R & GAATCCTTCAAAGGAATTGGAT & 60 \\
Comt-R1 & ACACTAACAAACATGTCACCAC & 60 \\
Comt-R2 & GCTTCGTAGCAGTTCTTCAACA & 60 \\
Comt-R3 & TACTCTTTGTGGCAGCTGATGT & 60 \\
Comt-R4 & CTGCTAAACCAACCCCAGTTAG & 60 \\
del-F3 & GGTGCTACTGTGAACATGATTGTCTCC & 67 \\
del-R4 & CAGTCGCCCAGGTAGTGGTATAAGAG & 67 \\
\hline
\end{tabular}

Buckinghamshire, UK). The Comt1 genomic region was amplified using Comt1-F and Comt1-R primer sets, and Comt2 was amplified using Comt2-F and Comt2-R primer sets (Table 1). A Straight Walk kit (BEX Co., Ltd., Tokyo, Japan) was used for Comt1 genome walking. PCR, electrophoresis, and sequencing were performed as described above.

\subsection{Sequence Analysis}

The Comt 1 and Comt 2 nucleic acid sequences were translated to their corresponding peptide sequences using EMBOSS Transeq (The European Bioinformatics Institute) [23]. BLAST (National Center for Biotechnology Information) [24] was used to search for similar sequences in the GenBank database. The sequences were aligned using Clustal W and a phylogenetic tree was generated using a Molecular Evolutionary Genetic Analysis software ver. 6.0 with 1000 bootstrap replicates [25].

\section{Results}

\subsection{Cloning of Comt1 and Comt2 from Habanero}

Comt1-F and Comt1-R primer sets and Comt2-F and Comt2-R primer sets were used for cloning the two OMTs from Habanero (C. chinense) placenta tissue. Two OMT sequences were cloned from Habanero and named Comt 1 and Comt2 in this study. Both Comt 1 and Comt2 had 1080 bp coding regions (CDS), and had 92\% similarity in nucleic acid sequence. Comt 1 had 99\% similarity with Comt (AF081214.1), and Comt2 had 99\% similarity with CaOMT1 (U83789.1). Sequencing the genomic regions of Comt 1 and Comt2 clarified that both genes had four exons, and the CDS comprised 407, 311, 65, and $300 \mathrm{bp}$. In contrast to the exons, the sequences and the length of the introns differed between the two genes. Comt 1 consisted of three introns, each comprising 241, 105, and 423 bp, whereas Comt 2 comprised 1164, 251, and 440 bp. The predicted protein sequences were 359 amino acids for COMT1 and COMT2. The two protein sequences showed 88\% residue identity (315/359), and showed high similarity with other OMTs, particularly with COMTs reported from other plants species (Figure 2).

\subsection{Phylogenetic Analysis of COMT1 and COMT2}

A phylogenetic tree was constructed with COMT1, COMT2, and SAM methyltransferases (SAM-Mtases) of other plant species (Figure 3). Twenty-nine SAM-Mtases were clustered into three groups: group 1 comprised COMT, chalcone OMT (CHOMT), and catechol OMT (CTOMT); group 2 comprised various SAM-Mtases including myricetin OMT (MOMT), flavonoid OMT (FOMT), and isoflavone OMT (IOMT); group 3 comprised jasmonic acid carboxyl methyltransferase (JAMT), salicylic acid carboxyl methyltransferase (SAMT), and indole-3-acetate OMT (IAOMT). COMT1 and COMT2 were located close to group 1. 


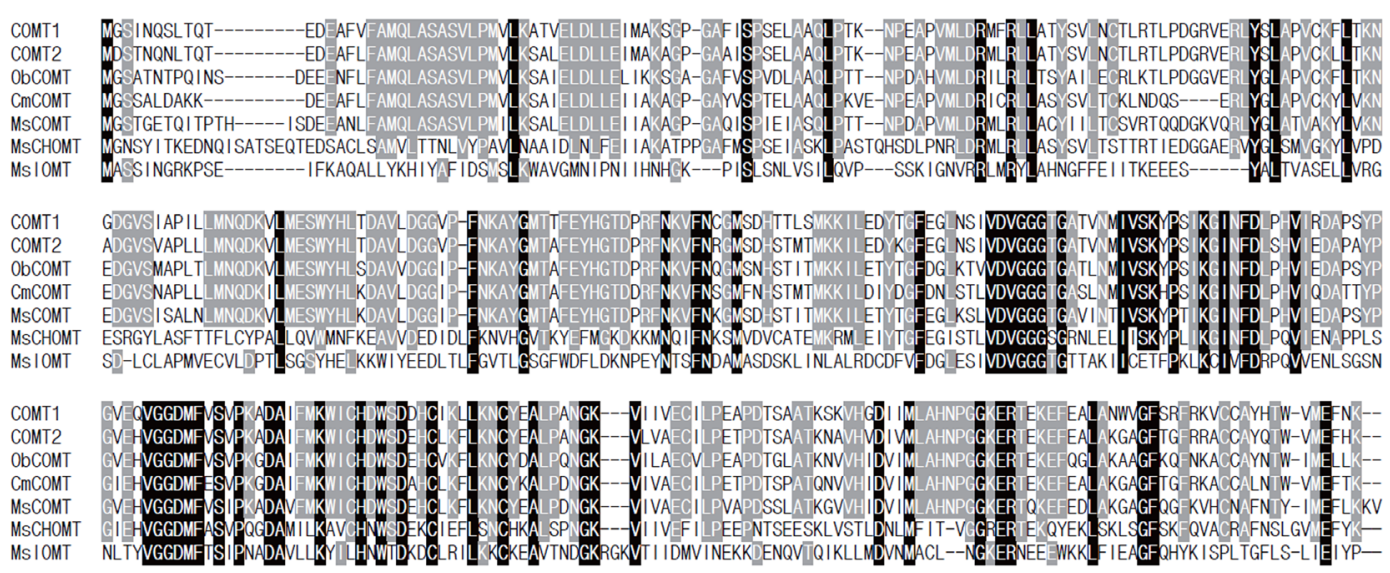

Figure 2. Alignment of the deduced amino acid sequences of Habanero caffeic acid $O$-methyltransferases (COMTs) with similar plant sequences. COMT1 and COMT2 of Habanero were aligned to ObCOMT (Ocimum basilicum, AAD38189.1), CmCOMT (Chrysanthemum $\times$ morifolium, BAK42963.1), MsCOMT (Medicago sativa, AAB46623.1), MsCHOMT (M. sativa, AAB4334159.1), and MsIOMT (M. sativa, AAC 49927.1). Shaded black indicates the nucleic acids conserved in all OMTs. Shaded gray indicates the nucleic acids conserved in all COMTs.

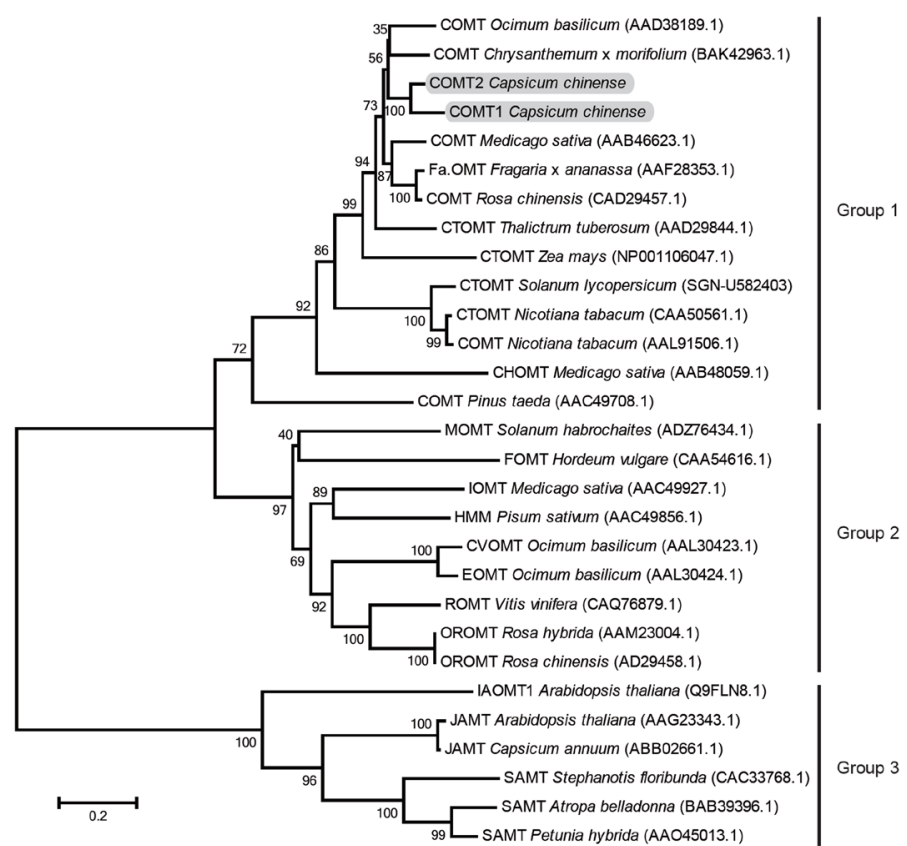

Figure 3. Phylogenetic relationships of Habanero caffeic acid $O$-methyltransferases (COMTs) with S-adenosyl-L-methionine methyltransferases (SAM-Mtases) of other plant species. A neighbor-joining tree comparing Habanero COMTs to the 27 SAM-Mtases. Three groups were present in the resulting tree: group 1 comprised COMT, chalcone OMT (CHOMT), and catechol OMT (CTOMT); group 2 comprised various SAM-Mtases including myricetin OMT (MOMT), flavonoid OMT (FOMT), and isoflavone OMT (IOMT); and group 3 comprised jasmonic acid carboxyl methyltransferase (JAMT), salicylic acid carboxyl methyltransferase (SAMT), and indole-3-acetate OMT (IAOMT). Branch labels include enzyme name, source plant, and GenBank protein ID. Bootstrap values are shown along the branches (from 1000 replicates).

\subsection{Sequence Comparison of Critical Amino-Acid Residues of the OMTs}

In the present study, 18 critical amino-acid residues of the SAM-binding domain, the catalytic domain, and the substrate binding domain were compared between 16 OMTs of groups 1 and 2 (Figure 4). Five of eight aminoacid residues in the SAM-binding domain were conserved in all OMTs, and all amino-acid residues were con- 


\begin{tabular}{|c|c|c|c|c|c|c|c|c|c|c|c|c|c|c|c|c|c|c|c|}
\hline & & \multicolumn{8}{|c|}{ SAM binding domain } & \multicolumn{3}{|c|}{\begin{tabular}{|l|} 
Catalytic domain \\
\end{tabular}} & \multicolumn{7}{|c|}{ Substrate binding domain } \\
\hline & & 202 & 204 & 227 & 228 & 247 & 248 & 261 & 267 & 265 & 293 & 325 & 126 & 172 & 176 & 310 & 315 & 316 & 319 \\
\hline \multirow[t]{8}{*}{ Group 1} & COMT1 & $D$ & $G$ & $D$ & $\mathrm{~L}$ & $D$ & $\mathrm{M}$ & $\mathrm{K}$ & W & $\mathrm{H}$ & $E$ & $E$ & $\mathrm{M}$ & $F$ & $\mathrm{M}$ & $\mathrm{V}$ & 1 & $\mathrm{M}$ & $\mathrm{H}$ \\
\hline & COMT2 & D & G & D & L & D & M & $\mathrm{k}$ & W & $\mathrm{H}$ & E & E & M & $\mathrm{F}$ & M & $\mathrm{v}$ & V & M & $\mathrm{H}$ \\
\hline & COMT (BAK42963.1) & D & G & D & L & D & M & $\mathrm{k}$ & W & $\mathrm{H}$ & E & E & M & $\mathrm{F}$ & M & $\mathrm{v}$ & 1 & M & $\mathrm{H}$ \\
\hline & COMT (AAD38189.1) & D & G & D & L & D & M & $\mathrm{K}$ & W & $\mathrm{H}$ & E & E & M & $\mathrm{F}$ & M & $\mathrm{v}$ & 1 & M & $\mathrm{H}$ \\
\hline & \begin{tabular}{|l} 
COMT (CAD29457.1) \\
\end{tabular} & D & G & D & L & D & M & $\mathrm{K}$ & W & $\mathrm{H}$ & E & E & M & $\mathrm{F}$ & M & $\mathrm{v}$ & 1 & M & $\mathrm{H}$ \\
\hline & $\begin{array}{l}\text { CTOMT (AAD29844.1) } \\
\end{array}$ & D & G & D & L & D & M & $\mathrm{K}$ & W & $\mathrm{H}$ & E & E & M & $\mathrm{F}$ & M & $\mathrm{F}$ & v & M & $\mathrm{H}$ \\
\hline & \begin{tabular}{|l|l} 
CTOMT (NP001106047.1) \\
\end{tabular} & D & G & D & L & D & M & $\mathrm{k}$ & w & H & E & E & M & $\mathrm{F}$ & M & $\mathrm{F}$ & 1 & M & $\mathrm{H}$ \\
\hline & CHOMT (AAB48059.1) & D & G & D & L & D & M & $\mathrm{K}$ & W & $\mathrm{H}$ & E & E & $\mathrm{F}$ & $\mathrm{F}$ & M & $s$ & $\mathrm{~L}$ & M & $T$ \\
\hline \multirow[t]{8}{*}{ Group 2} & CVOMT (AAL30423.1) & $\mathrm{D}$ & G & $\mathrm{D}$ & $\bar{L}$ & $D$ & M & $\mathrm{K}$ & $\bar{W}$ & $\mathrm{H}$ & $\mathrm{D}$ & $E$ & $\mathrm{~V}$ & $\mathrm{~F}$ & M & $\bar{F}$ & M & $\bar{A}$ & s \\
\hline & EOMT (AAL30424.1) & D & G & D & L & D & M & $\mathrm{K}$ & W & $\mathrm{H}$ & D & E & v & $\mathrm{F}$ & M & $\mathrm{F}$ & M & A & c \\
\hline & OROMT (AAM23004.1 ) & D & G & D & L & D & M & K & w & H & D & E & A & $\mathrm{F}$ & M & $\mathrm{F}$ & M & $\mathrm{L}$ & A \\
\hline & ROMT (CAQ76879.1) & D & G & D & L & D & M & K & w & H & D & E & A & $\mathrm{F}$ & M & $\mathrm{F}$ & M & $T$ & I \\
\hline & IOMT (AAC49927.1) & D & G & D & L & D & M & K & w & H & D & E & C & $\mathrm{F}$ & M & M & V & N & C \\
\hline & HMM (AAC49856.1) & D & A & D & L & D & M & K & w & H & D & E & G & $\mathrm{F}$ & M & Y & v & v & $T$ \\
\hline & MOMT (ADZ76434.1) & D & G & D & $\mathrm{R}$ & s & M & $\mathrm{k}$ & W & $\mathrm{H}$ & D & E & $\mathrm{F}$ & $\mathrm{F}$ & M & M & L & 1 & I \\
\hline & FOMT (CAA54616.1) & D & A & D & Q & D & M & $\mathrm{K}$ & W & H & D & $\mathrm{V}$ & L & $\mathrm{F}$ & M & M & L & $s$ & M \\
\hline
\end{tabular}

Figure 4. Comparison of the critical amino acid residues for $O$-methyltransferases (OMTs). Critical amino acid residues for the S-adenosyl-L-methionine (SAM) binding domain, the catalytic domain, and the substrate binding domain were chosen according to Zubieta et al. [20]. The shaded box indicates the residues conserved in at least two different proteins.

served in group 1. The catalytic domain was also highly conserved in all OMTs, and all amino-acid residues were conserved in group 1. In contrast, only two (Phe-172 and Met-176) of seven amino-acid residues were conserved in the substrate binding domain of all OMTs, and the other five residues varied. In particular, group 2 OMT amino-acid residues were highly variable, suggesting that the difference in these amino-acid residues is critical for substrate specificity. The amino-acid residues were highly conserved in group 1 compared to group 2, and all residues in the COMTs were conserved except for Val-315 of COMT2.

\subsection{Comt1 and Comt2 Expression Analysis}

The Comt 1 and Comt2 expression analysis was conducted with RNA samples derived from the placenta tissue (stage 1: immature green, stage 2: mature green and stage 3: mature red) (Figure 5(a)), leaf, stem, and root by RT-PCR. The accumulation of capsaicinoids in Habanero increased drastically beginning at stage 2 (Figure 5(b)). Comt 1 and Comt 2 showed the same expression pattern in placenta tissue. Both genes were highly expressed during stages 1 and 2 and then decreased during stage 3 (Figure 5(c)). Although Comt 1 expression was not detected in leaf, stem, or root, Comt2 was highly expressed in these tissues (Figure 5(c)).

\subsection{Analysis of Comt1 Deletion Mutants}

Abraham-Juarez et al. [6] silenced Comt (AF081214.1) by VIGS, which has 99\% similarity with Comt1, and showed that the accumulation of capsaicinoids decreased drastically. This result indicates that Comt loss-offunction mutation could induce loss of pungency in Capsicum. No.3341 (C. chinense) is a non-pungent cultivar found in our previous study that Pun1 and p-AMT could not account for its non-pungency [26]. No expression was detected when Comt1-F was designed to anneal the 5' untranslated region (UTR) and Comt1-R was designed to anneal the 3'UTR primer set for detecting Comt1 in RNA samples derived from the placenta of No.3341 (Figure 6). As it was possible that primers could not anneal to the cDNA of No.3341 because of single nucleotide polymorphisms (SNPs) located at the annealing position, an additional four reverse primers were designed and used for PCR. Comt1 was detected by all the primer sets in Habanero (Figure 6). In contrast, only Comt-R1, which was designed at almost the middle of the coding region, could detect the Comt1 transcript in No.3341 (Figure 6). The same result was obtained when genomic PCR was conducted using the same primers (Figure 6).

The race-method was used to obtain the full sequence, because expression of a truncated Comt 1 transcript was suggested in No.3341. Sequencing the full-length Comt 1 cDNA of No.3341 clarified that the truncated transcript was expressed. The predicted protein sequences were 283 amino acids for COMT1 of No.3341 compared to 359 amino acids for Habanero (Figure 7). COMT1 of No.3341 differed from 262 amino acids residue compared to 


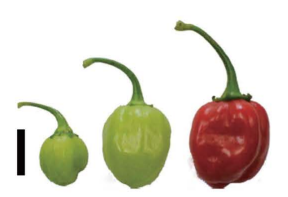

(a)

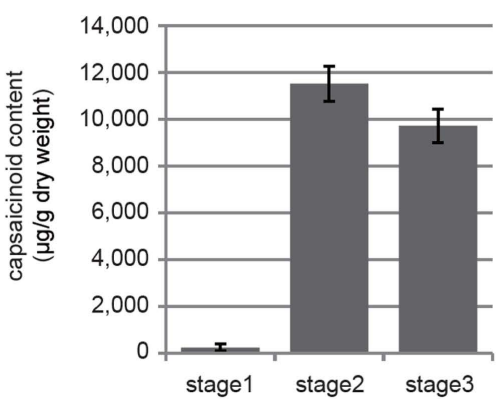

(b)

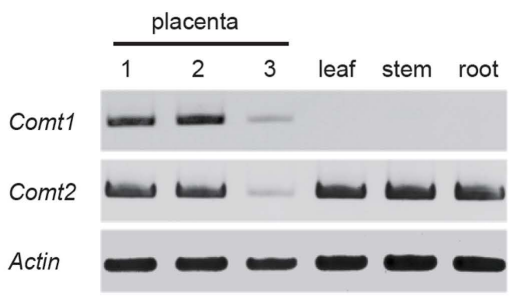

(c)

Figure 5. Assessment of capsaicinoid accumulation and expression analysis of caffeic acid $O$-methyltransferases (Comts) in Habanero. (a) The Habanero fruit developmental stage used for the study (stage 1: immature green, stage 2: mature green and stage 3: mature red from left to right). Bar indicates $2 \mathrm{~cm}$; (b) Capsaicinoid content at each Habanero fruit stage. Error bars: standard deviation for results of three plants. (c) Comt1 and Comt2 expression in placenta tissue (stage 1-3), leaf, stem, and root of Habanero. Actin was used as the positive internal control.
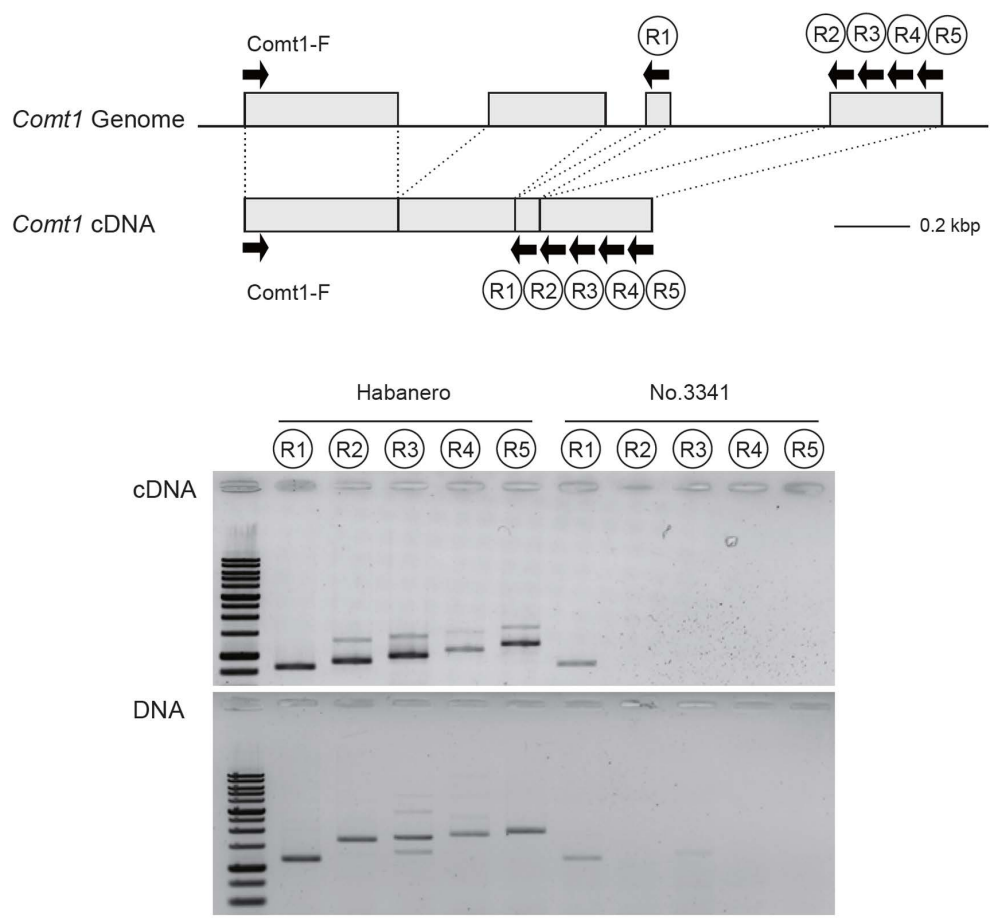

Figure 6. Genomic and cDNA structures of caffeic acid $O$-methyltransferase 1 (Comt 1 ), and electrophoresis of polymerase chain reaction amplified fragments. Expression of the truncated Comt 1 transcript was suggested in No.3341. R1, R2, R3, R4, and R5 indicate Comt-R1, Comt-R2, Comt-R3, Comt-R4, and Comt1-R, respectively.

Habanero, lacking almost half of the $O$-methyltransferase domain. The genomic region was sequenced by genome walk and clarified that Comt 1 of No.3341 possessed a $2.3 \mathrm{kbp}$ large deletion from the 5' region of the fourth exon to the intergenic region (Figure 8).

The co-dominant DNA markers del-F3 and del-R4 were designed to confirm the relationship between the Comt1 deletion mutation and non-pungency in No.3341 (Table 1). The pungent and non-pungent phenotype segregated 3:1 in the $\mathrm{F}_{2}$ population obtained by crossing Habanero with No.3341 $(\mathrm{n}=98$, chi-square $=0.340, P$ value $=0.560$ ). Contrary to our expectations, the Comt 1 mutation did not co-segregate with non-pungency of No.3341 (Figure 9(a)). Thus, the Comt1 mutation was not related to non-pungency of No.3341. This result was confirmed by screening several pungent accessions harboring the same Comt1 deletion mutation (Figure 9(b)). 
Habanero MGSINQSLTQTEDEAFVFAMQLASASVLPMVLKATVELDLLE IMAKSGPGAF I SPSELAAQLPTKNPEAPVML No.3341 MGSINESLTETEDEAFVFAMELASASVLPMVLKATVELDLLE I MAKSGPGAF I SPSELAAQLPTKNPEAPVML

Habanero DRMFRLLATYSVLNCTLRTLPDGRVERLYSLAPVCKFLTKNGDGVSI API LLIMNQDKVLMESWYHLTDAVLDG No.3341 DRMFRLLATYSVLNCTLRTLPDGRVERLYSLAPVCKFLTKNGDGVS I API LLMNQDKVLMESWYHLTDAVLDG

Habanero GVPFNKAYGMTTFEYHGTDPRFNKVFNCGMSDHTTLSMKK I LEDYTGFEGLNS I VDVGGGTGATVNM I VSKYP No.3341 GVEFNKAYGMTTFEYHGTDPRFNKVFNCGMSDHTTLFMKK I LEDYTGFEGLNS IVDVGGGTGATVNM I VSKYP Habanero SIKG I NFDLPHVIRDAPSYPGVEQVGGDMFVSVPKADA IFMKWI CHDWSDDHC I KLLKNCYEALPANGKV I IV No.3341 SIKGINFDLPHVIRDAPSYPGVEQVGGDMFVSVPKADA IFMKVMPIELPTVSPTSPGFRYVEVE---_

Habaner o ECILPEAPDTSAATKSKVHGDI IMLAHNPGGKERTEKEFEALANWVGFSRFRKVCCAYHTWVMEFNK No. 3341

Figure 7. Alignment of the deduced amino acid sequence of caffeic acid $O$-methyltransferase 1 (COMT1) from Habanero and No.3341. COMT1 of Habanero and No.3341 were aligned. The underlined part indicates the $O$-methyltransferase domain. A truncated COMT1 was produced in No.3341 because of the $2.3 \mathrm{kbp}$ genetic mutation. Shaded gray for No.3341 indicates the amino acid residues that differ from Habanero. Shaded black indicates the critical amino acid residues of the S-adenosyl-L-methionine (SAM) binding domain, catalytic domain, and substrate binding domain. Triangle indicates the His-265 necessary for catalytic activity.

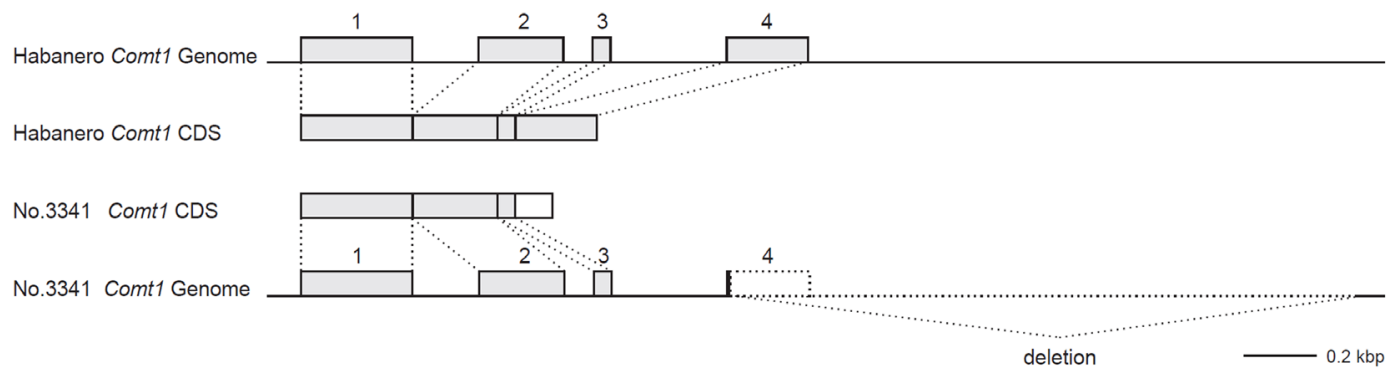

Figure 8. Genomic and coding region structures of caffeic acid $O$-methyltransferase 1 (Comt 1 ) for Habanero and No.3341. Boxes indicate Comt1 exons. The location of the $2.3 \mathrm{kbp}$ deletion is shown in the Comt 1 No.3341 genomic structure.

\section{Discussion}

\subsection{Comt1 and Comt2 Are Different Genes Coded in Different Loci}

The CaOMT1 (U83789.1) from C. annuum [21] and Comt (AF081214.1) from C. chinense [7] are two OMTs expressed in placenta tissue that have been cloned previously. Because CaOMT1 (U83789.1) and Comt (AF081214.1) were cloned by different research teams and from different Capsicum species, it is unclear whether the two transcripts are allelic or genes coded by different loci. In the present study, two transcripts were cloned from the Habanero inbred line. Comt1, cloned from Habanero, had 99\% similarity with Comt (AF081214.1) and Comt2 had 99\% similarity with CaOMT1 (U83789.1). In addition, the length and sequences of the Comt1 and Comt2 introns differed. Moreover, whole genome sequencing of Capsicum clarified that Comt has undergone extensive gene duplication [27]. Searching the whole genome sequence of Comt1 and Comt2 revealed that the two genes are coded in chromosome 3 with a 13 kbp distance. Thus, it can be concluded that Comt 1 and Comt 2 are not allelic but different genes coded by different loci.

\subsection{Comt1 and Comt2 are Candidate Genes for Capsaicinoid Biosynthesis}

Plant SAM-Mtases are key enzymes in phenylpropanoid, flavonoid, and many other metabolic pathways [28]. OMT is one of the SAM-Mtases and can be classified into two groups, a group including COMT and another group that includes other OMTs with various substrate specificities [19] [28]-[30]. In the present study, a phylogenetic analysis of 29 SAM-Mtases was conducted and showed that two Habanero OMTs were located closely with COMTs in group 1 of other plant species (Figure 3). These results suggest that COMT1 and COMT2 have similar substrate preferences to COMTs of other plant species. 


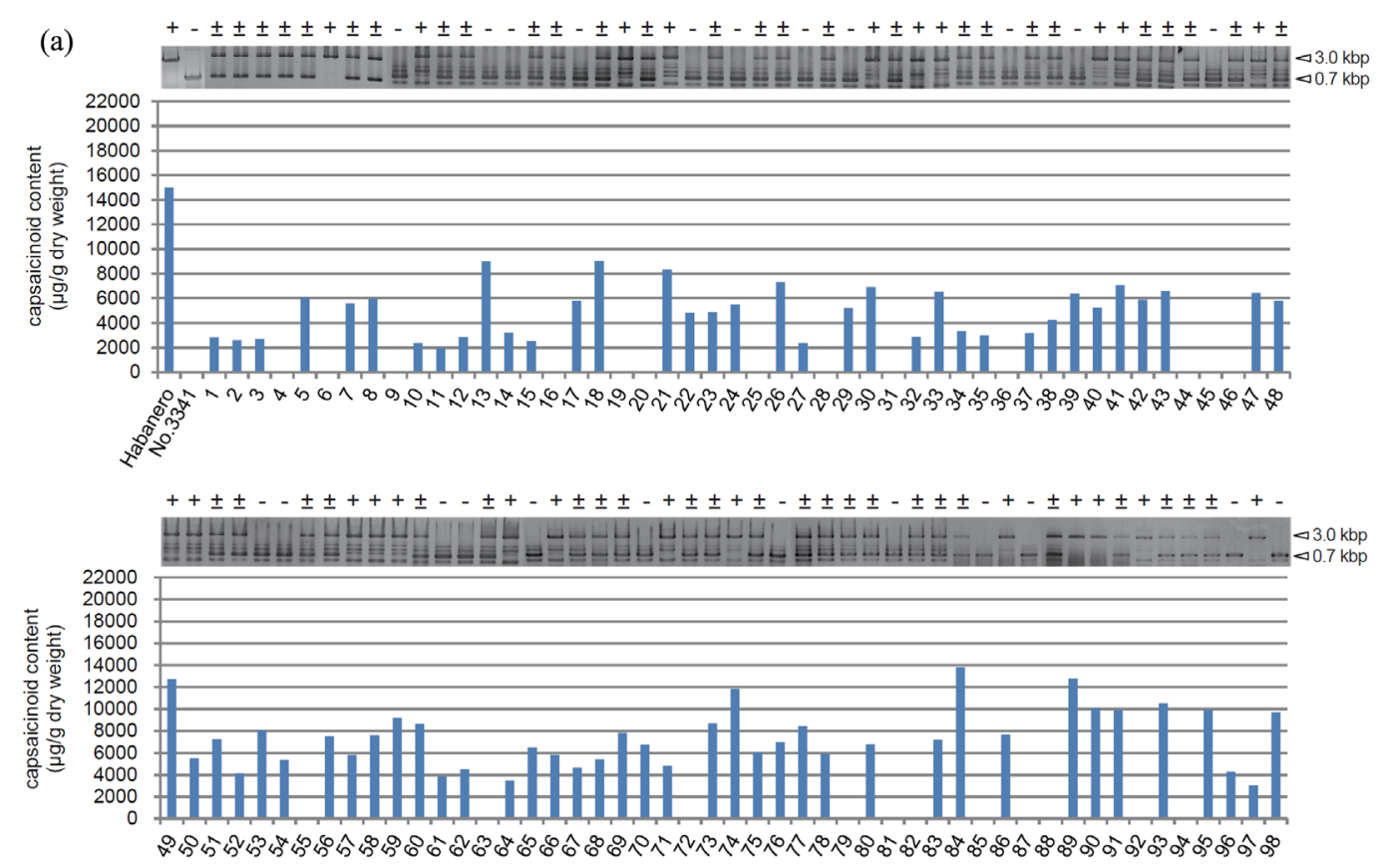

(b)

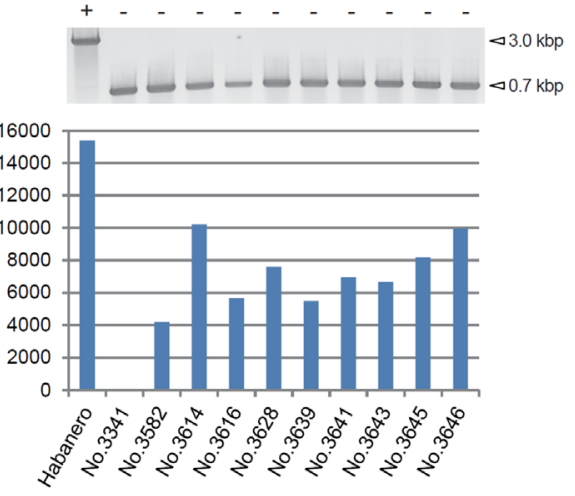

Figure 9. DNA marker analysis. (a) The DNA marker pattern of caffeic acid O-methyltransferase 1 (Comt1) did not co-segregate with the pungency trait in $\mathrm{F}_{2}$ individuals. The capsaicinoid contents measured by high performance liquid chromatography are expressed in $\mu \mathrm{g} \cdot \mathrm{g}^{-1}$ dry weight. The gels show DNA marker patterns of the $\mathrm{F}_{2}$ individuals. + and - indicates individuals homozygous in the Habanero type ( $\left.3 \mathrm{kbp}\right)$ and the No. 3341 type (0.7 kbp), respectively, and \pm indicates a heterozygote. (b) Pungency phenotype in Comt1-deletion mutants.

Zubietaet al. [20] studied the precise structural basis of enzyme activity using CHOMT and IOMT of Medicago sativa (alfalfa). Plant OMTs use SAM as a methyl source, yielding S-adenosyl-L-homocysteine and the methyl ether derivatives as products. Critical residues for the SAM-binding domain and the catalytic domain were highly conserved in the 16 OMTs clustered in groups 1 and 2 of our phylogenetic tree (Figure 3 and Figure 4). In contrast, substrate binding domain diversity was observed for critical residues (Figure 4). As sequential diversity was observed particularly in the OMTs of group 2, these differences seemed to reflect substrate specificity. Six out of seven residues among the five COMTs of group 1 were conserved, suggesting that these OMTs use the same substrate. However, as amino acid 315 was a valine only in COMT2, but an isoleucine in other COMTs, further enzymological study is needed to clarify how this difference affects substrate preference.

Capsaicinoids start to accumulate in Capsicum beginning 20 days post-anthesis [11], which coincides with the accumulation pattern observed in Habanero (Figure 5(b)). As Comt 1 and Comt2 are expressed in placenta tissue where the capsaicinoids are synthesized and expressed prior to capsaicinoid accumulation, the two genes could be candidates for capsaicinoid biosynthesis (Figure 5(c)). The expression of the two genes differed in other or- 
gans. Only Comt2 was expressed in the leaf, stem, and root (Figure 5(c)), suggesting that Comt2 has broad functions in addition to capsaicinoid synthesis. Considering gene duplication of Comt [27] and our phylogenetic analysis (Figure 3), Comt1 may have been duplicated from Comt2.

\subsection{The Comt1 Loss-of-Function Mutation Does Not Affect Capsaicinoid Biosynthesis in Capsicum}

Based on our phylogenetic and expression analyses, Comt 1 and Comt2 could be candidates for capsaicinoid biosynthesis. Because Abraham-Juarez et al. [6] silenced Comt (AF081214.1) by VIGS, which had 99\% similarity with Comt1, and showed that the accumulation of capsaicinoids decreases drastically, it was assumed that the Comt loss-of-function mutation would cause a loss of pungency in Capsicum. As transformation of Capsicum is technically difficult [31], a natural mutant of a specific gene is valuable for predicting gene function. In the present study, non-pungent No.3341 was screened for the expression of the truncated Comt 1 transcript which was caused by the large deletion mutation in the genomic region (Figure 8), lacking almost half of the OMT domain. Zubieta et al., [20] have clearly shown that histidine in CHOMT (His-278) catalytic domain and IOMT (His-257) are absolutely necessary catalytic residues. The predicted COMT1 of No.3341 lacks His-265 due to a mutation, which would diminish enzyme activity. $\mathrm{F}_{2}$ populations obtained from crossing No.3341 and Habanero were prepared to elucidate the relationship between mutated Comt 1 and capsaicinoid synthesis. Contrary to our expectations, the Comt1 mutation did not co-segregate with the non-pungency trait (Figure 9(a)). With our previous study [26], it was shown that loss of pungency in No.3341 is controlled by a single recessive gene that is neither Pun1, p-AMT nor Comt1. For further confirmation, Comt1 mutants were screened from the Capsicumgermplasm and nine pungent accessions harboring the same Comt1 deletion mutation were screened (Figure 9(b)). Thus it was concluded that Comt1 loss-of-function mutation is insufficient for loss of pungency in Capsicum. Because Comt1 loss-of-function mutants were frequently found, those might have been selected artificially for other valuable traits caused by mutation of Comt1. Further study is needed to clarify this point.

In the present study, Comt 1 and Comt 2 were cloned from the Habanero pungent pepper (C. chinense). Moreover, an analysis of Comt1-mutated peppers clarified that Comt1 loss-of-function mutation was insufficient for loss of pungency. In contrast, Abraham-Juarez et al. [6] silenced Comt (AF081214.1) by VIGS, which had 99\% similarity with Comt1, and showed that the accumulation of capsaicinoids decreased drastically. Expression of Comt2 in placenta tissue must be considered to explain these contradictory findings. In the study of Abraham Juarez et al. [6], Comt(Comt1)-silenced plants had undetectable levels of Comt mRNA or a reduction of an 8 24 folds by northern blotting and RT-PCR. Our results showed that the Comt1 and Comt2 sequence similarity was $92 \%$ in the coding regions. Thus, even Comt1 was specifically silenced by VIGS, Comt 2 would still be detected by the DNA probes or primers designed to anneal coding regions of Comt1. Two possibilities should be discussed to explain these results. When two or more genes have high sequence similarity, a specific sequence of siRNA induces simultaneous silencing such as in petunia, Trenia plants, yellow beans (Glycine max L.), and dahlia [32]-[37]. Silencing of Comt1 by VIGS may have simultaneously silenced Comt2; thus, leading to decrease capsaicinoid accumulation. The second possibility is that serrano chili pepper (C. annuum cv. Tampiqueño 74) used in the study of Abraham Juarez et al. [6] had a Comt2 expression defect, such as the deletion of the promoter region. If Comt 1 was silenced in such a pepper plant, both Comt 1 and Comt 2 would not function and capsaicinoid accumulation would decrease drastically. In the present study, 280 pepper germplasms were screened for a Comt2 loss-of-function mutant, which will be valuable for asking the relation of Comts to capsaicinoid synthesis, using high-throughput sequencing technology, but no mutant was found (data not shown). It can be concluded that the Comt1 mutation is insufficient for the loss of pungency in Capsicum. Participation of COMT in phenylpropanoid-mediated capsaicinoid biosynthesis has long been proposed [5] [7] [18] [19]. Mozourek et al. [9] proposed the participation of caffeoyl-CoA O-methyltransferase (CCoAOMT) instead of COMT in the phenylpropanoid pathway of capsaicinoid biosynthesis. Thus, further study is needed to clarify which genes are critically important for capsaicinoid biosynthesis and could be a target for controlling fruit pungency in Capsicum.

\section{GenBank Accession Numbers}

CDS sequence of Comt1 (AB971124), Comt2 (AB971126), genomic sequence of Comt1 (AB971125), and Comt2 (AB971127) of Habanero. CDS sequence of Comt1 (LC050124) and genomic sequence of Comt1 
(LC050125) of No. 3341.

\section{Acknowledgements}

We thank Toshio Sakakibara, Masaru Matsuda, and Koji Nishikawa (Experimental Farm, Kyoto University) for technical assistance with the field experiment. Motoaki Doi, Munetaka Hosokawa, Keisuke Katsura, Takashi Kawai, Daiki Matsumoto, Tetsuya Nakazaki, Sho Ohno, and Hiroki Saito (Kyoto University) are appreciated for their useful discussion. This work was supported by a Grant-in-Aid for Young Scientists (B) [25850018] from the Japan Society for the Promotion of Science.

\section{References}

[1] Singh, R.J. (2007) Genetic Resources, Chromosome Engineering, and Crop Improvement. Vol. 3. Taylor and Francis CRC Press, Boca Raton.

[2] Perry, L., Dickau, R., Zarrillo, S., Holst, I., Pearsall, D.M., Piperno D.R., Berman, M.J., Cooke, R.G., Rademaker, K., Ranere, A.J., Raymond, J.S., Sandweiss, D.H., Scaramelli, F., Tarble, K. and Zeidler, J.A. (2007) Starch Fossils and the Domestication and Dispersal of Chili Peppers (Capsicum spp. L.) in the Americas. Science, 315, 986-988. http://dx.doi.org/10.1126/science.1136914

[3] Leete, E. and Louden, M. (1968) Biosynthesis of Capsaicin and Dihydrocapsaicin in Capsicum frutescens. Journal of the American Chemical Society, 90, 6837-6841. http://dx.doi.org/10.1021/ja01026a049

[4] Suzuki, T., Kawada, T. and Iwai, K. (1981) Biosynthesis of Acyl Moieties of Capsaicin and Its Analogues from Valine and Leucine in Capsicum Fruits. Plant and Cell Physiology, 22, 23-32.

[5] Sukrasno, N. and Yeoman, M.M. (1993) Phenylpropanoid Metabolism during Growth and Development of Capsicum frutescens Fruits. Phytochemistry, 32, 839-844. http://dx.doi.org/10.1016/0031-9422(93)85217-F

[6] Abraham-Juarez, M.D., Rocha-Granados, M.D., López, M.G., Rivera-Bustamante, R.F. and Ochoa-Alejo, N. (2008) Virus-Induced Silencing of Comt, pAmt and Kas Genes Results in a Reduction of Capsaicinoid Accumulation in Chili Pepper Fruits. Planta, 227, 681-695. http://dx.doi.org/10.1007/s00425-007-0651-7

[7] Curry, J., Aluru, M., Mendoza, M., Nevarez, J., Melendrez, M. and O’Connell, M.A. (1999) Transcripts for Possible Capsaicinoid Biosynthetic Genes Are Differentially Accumulated in Pungent and Non-Pungent Capsicum spp. Plant Science, 148, 47-57. http://dx.doi.org/10.1016/S0168-9452(99)00118-1

[8] Aluru, M.R., Mazourek, M., Landry, L.G., Curry, J., Jahn, M. and O’Connell, M.A. (2003) Differential Expression of Fatty Acid Synthase Genes, Acl, Fat and Kas in Capsicum Fruit. Journal of Experimental Botany, 54, 1655-1664. http://dx.doi.org/10.1093/jxb/erg176

[9] Mazourek, M., Pujar, A., Borovsky, Y., Paran, I., Mueller, L. and Jahn, M.M. (2009) A Dynamic Interface for Capsaicinoid Systems Biology. Plant Physiology, 150, 1806-1821. http://dx.doi.org/10.1104/pp.109.136549

[10] Boswell, V.R. (1937) Improvement and Genetics of Tomatoes, Peppers, and Eggplant. In: Wallace, H.A., Ed., Yearbook of Agriculture, United States Government Printing Office, Washington DC, 176-206.

[11] Stewart, C., Kang, B.C., Liu, K., Mazourek, M., Moore, S.L., Yoo, E.Y., Kim, B.D., Paran, I. and Jahn, M.M. (2005) The Pun1 Gene for Pungency in Pepper Encodes a Putative Acyltransferase. Plant Journal, 42, 675-688. http://dx.doi.org/10.1111/j.1365-313X.2005.02410.x

[12] Lang, Y.Q., Kisaka, H., Sugiyama, R., Nomura, K., Morita, A., Watanabe, T., Tanaka, Y., Yazawa, S. and Miwa, T. (2009) Functional Loss of pAMT Results in Biosynthesis of Capsinoids, Capsaicinoid Analogs, in Capsicum annuum cv. CH-19 Sweet. Plant Journal, 59, 953-961. http://dx.doi.org/10.1111/j.1365-313X.2009.03921.x

[13] Tanaka, Y., Hosokawa, M., Miwa, T., Watanabe, T. and Yazawa, S. (2010) Newly Mutated Putative-Aminotransferase in Nonpungent Pepper (Capsicum annuum) Results in Biosynthesis of Capsinoids, Capsaicinoid Analogues. Journal of Agricultural and Food Chemistry, 58, 1761-1767. http://dx.doi.org/10.1021/jf903282r

[14] Stellari, G.M., Mazourek, M. and Jahn, M.M. (2010) Contrasting Modes for Loss of Pungency between Cultivated and Wild Species of Capsicum. Heredity, 104, 460-471. http://dx.doi.org/10.1038/hdy.2009.131

[15] Tanaka, Y., Hosokawa, M., Miwa, T., Watanabe, T. and Yazawa, S. (2010) Novel Loss-of-Function Putative Aminotransferase Alleles Cause Biosynthesis of Capsinoids, Nonpungent Capsaicinoid Analogues, in Mildly Pungent Chili Peppers (Capsicum chinense). Journal of Agricultural and Food Chemistry, 58, 11762-11767. http://dx.doi.org/10.1021/jf1019642

[16] Koeda, S., Sato, K., Tomi, K., Tanaka, Y., Takisawa, R., Hosokawa, M., Doi, M., Nakazaki, T. and Kitajima, A. (2014) Analysis of Non-Pungency, Aroma, and Origin of a Capsicum chinense Cultivar from a Caribbean Island. Journal of the Japanese Society for Horticultural Science, 83, 244-251. http://dx.doi.org/10.2503/jishs1.CH-105 
[17] Fujiwake, H., Suzuki, T. and Iwai, K. (1982) Intracellular Distribution of Enzymes and Intermediates Involved in Biosynthesis of Capsaicin and Its Analogues in Capsicum Fruits. Agricultural and Biological Chemistry, 46, 2685-2689. http://dx.doi.org/10.1271/bbb1961.46.2685

[18] Fujiwake, H., Suzuki, T. and Iwai, K. (1982) Capsaicinoid Formation in the Protoplast from Placenta of Capsicum Fruits. Agricultural and Biological Chemistry, 46, 2591-2592. http://dx.doi.org/10.1271/bbb1961.46.2591

[19] Gang, D.R., Lavid, N., Zubieta, C., Chen, F., Beuerle, T., Lewinsohn, E., Noel, J.P. and Pichersky, E. (2002) Characterization of Phenylpropene $O$-Methyltransferases from Sweet Basil: Facile Change of Substrate Specificity and Convergent Evolution within a Plant $O$-Methyltransferase Family. The Plant Cell Online, 14, 505-519. http://dx.doi.org/10.1105/tpc.010327

[20] Zubieta, C., He, X.-Z., Dixon, R.A. and Noel, J.P. (2001) Structures of Two Natural Product Methyltransferases Reveal the Basis for Substrate Specificity in Plant O-Methyltransferase. Nature Structural and Molecular Biology, 8, 271279. http://dx.doi.org/10.1038/85029

[21] Lee, B., Choi, D. and Lee, K.W. (1998) Isolation and Characterization of $O$-Diphenol-O-Methyltransferase cDNA Clone in Hot Pepper (Capsicum annuum L.). Journal of Plant Biology, 41, 9-15. http://dx.doi.org/10.1007/BF03030467

[22] Koeda, S., Hosokawa, M., Saito, H. and Doi, M. (2013) Temperature-Sensitive Phenotype Caused by Natural Mutation in Capsicum Latescent in Two Tropical Regions. Journal of Plant Research, 126, 675-684. http://dx.doi.org/10.1007/s10265-013-0564-4

[23] Rice, P., Longden, I. and Bleasby, A. (2000) EMBOSS: The European Molecular Biology Open Software Suite. Trends in Genetics, 16, 276-277. http://dx.doi.org/10.1016/S0168-9525(00)02024-2

[24] Altschul, S.F., Gish, W., Miller, W., Myers, E.W. and Lipman, D.J. (1990) Basic Local Alignment Search Tool. Journal of Molecular Biology, 215, 403-410. http://dx.doi.org/10.1016/S0022-2836(05)80360-2

[25] Tamura, K., Stecher, G., Peterson, D., Filipski, A. and Kumar, S. (2013) MEGA6: Molecular Evolutionary Genetics Analysis Version 6.0. Molecular Biology and Evolution, 30, 2725-2729. http://dx.doi.org/10.1093/molbev/mst197

[26] Koeda, S., Sato, K., Takisawa, R. and Kitajima, A. (2015) Inheritance of the Non-Pungency in 'No.3341' (Capsicum chinense). The Horticulture Journal, in Press. http://dx.doi.org/10.2503/hortj.MI-049

[27] Kim, S., Park, M., Yeom, S., Kim, Y.-M., Lee, J.M., Lee, H.-A., Seo, E., Choi, J., Cheong, K., Kim, K.-T., Jung, K., Lee, G.-W., Oh, S.-G., Bae, C.-Y., Kim, S.-B., Lee, H.-Y., Kim, S.-Y., Kim, M.-S., Kang, B.-C., Jo, Y.D., Yang, H.-B., Jeong, H.-J., Kang, W.-H., Kwon, J.-K., Shin, C., Lim, J.Y., Park, J.H., Huh, J.H., Kim, J.-S., Kim, B.-D., Cohen, O., Paran, I., Suh, M.C., Lee, S.B., Kim, Y.-K., Shin, Y., Noh, S.-J., Park, J., Seo, Y.S., Kwon, S.-Y., Kim, H.A., Park, J.M., Kim, H.-J., Choi, S.-B., Bosland, P.W., Reeves, G., Jo, S.-W., Lee, B.-W., Cho, H.-T., Choi, H.-S., Lee, M.-S., Yu, Y., Choi, Y.D., Park, B.-S., van Deynze, A., Ashrafi, H., Hill, T., Kim, W.T., Pai, H.-S., Ahn, H.K., Yeam, I., Giovannoni, J.J., Rose, J.K.C., Sørensen, I., Lee, S.-J., Kim, R.W., Choi, I.-Y., Choi, B.-S., Lim, J.-S., Lee, Y.-H. and Choi, D. (2014) Genome Sequence of the Hot Pepper Provides Insights into the Evolution of Pungency in Capsicum Species. Nature Genetics, 46, 270-278. http://dx.doi.org/10.1038/ng.2877

[28] Joshi, C.P. and Chiang, V.L. (1998) Conserved Sequence Motifs in Plant S-Adenosyl-L-Methionine-Dependent Methyltransferases. Plant Molecular Biology, 37, 663-674. http://dx.doi.org/10.1023/A:1006035210889

[29] Ibrahim, R.K., Bruneau, A. and Bantignies, B. (1998) Plant O-Methyltransferases: Molecular Analysis, Common Signature and Classification. Plant Molecular Biology, 36, 1-10. http://dx.doi.org/10.1023/A:1005939803300

[30] Scalliet, G., Journot, N., Jullien, F., Baudino, S., Magnard, J.L., Channelière, S., Vergne, P., Dumas, C., Bendahmane, M., Cock, J.M. and Hugueney, P. (2002) Biosynthesis of the Major Scent Components 3,5-Dimethoxytoluene and 1,3,5-Trimethoxybenzene by Novel Rose O-Methyltransferases. FEBS Letters, 523, 113-118. http://dx.doi.org/10.1016/S0014-5793(02)02956-3

[31] Kothari, S.L., Joshi, A., Kachhwaha, S. and Ochoa-Alejo, N. (2010) Chilli Peppers-A Review on Tissue Culture and Transgenesis. Biotechnology Advances, 28, 35-48. http://dx.doi.org/10.1016/j.biotechadv.2009.08.005

[32] Napoli, C., Lemieux, C. and Jorgensen, R. (1990) Introduction of a Chimeric Chalcone Synthase Gene into Petunia Results in Reversible Co-Suppression of Homologous Genes in Trans. The Plant Cell Online, 2, 279-289. http://dx.doi.org/10.1105/tpc.2.4.279

[33] Van Der Krol, A.R., Mur, L.A., Beld, M., Mol, J.N.M. and Stuitje, A.R. (1990) Flavonoid Genes in Petunia: Addition of a Limited Number of Gene Copies May Lead to a Suppression of Gene Expression. The Plant Cell Online, 2, 291299. http://dx.doi.org/10.1105/tpc.2.4.291

[34] Fukusaki, E.I., Kawasaki, K., Kajiyama, S., An, C.I., Suzuki, K., Tanaka, Y. and Kobayashi, A. (2004) Flower Color Modulations of Torenia hybrida by Downregulation of Chalcone Synthase Genes with RNA Interference. Journal of Biotechnology, 111, 229-240. http://dx.doi.org/10.1016/j.jbiotec.2004.02.019

[35] Kurauchi, T., Matsumoto, T., Taneda, A., Sano, T. and Senda, M. (2009) Endogenous Short Interfering RNAs of 
Chalcone Synthase Genes Associated with Inhibition of Seed Coat Pigmentation in Soybean. Breed Science, 59, 419426. http://dx.doi.org/10.1270/jsbbs.59.419

[36] Tuteja, J.H., Zabala, G., Varala, K., Hudson, M. and Vodkin, L.O. (2009) Endogenous, Tissue-Specific Short Interfering RNAs Silence the Chalcone Synthase Gene Family in Glycine max Seed Coats. Plant Cell, 21, 3063-3077. http://dx.doi.org/10.1105/tpc.109.069856

[37] Ohno, S., Hosokawa, M., Kojima, M., Kitamura, Y., Hoshino, A., Tatsuzawa, F., Doi, M. and Yazawa, S. (2011) Simultaneous Post-Transcriptional Gene Silencing of Two Different Chalcone Synthase Genes Resulting in Pure White Flowers in the Octoploid Dahlia. Planta, 234, 945-958. http://dx.doi.org/10.1007/s00425-011-1456-2 